\title{
Clinical utility gene card for: Transient Neonatal Diabetes Mellitus, 6q24-related
}

\author{
Deborah Mackay ${ }^{\star, 1,2}$, Susanne Bens ${ }^{3}$, Guiomar Perez de Nanclares ${ }^{4}$, Reiner Siebert ${ }^{3}$ and I Karen Temple ${ }^{1,5}$
}

European Journal of Human Genetics (2014) 22, doi:10.1038/ejhg.2014.27; published online 26 February 2014

\section{DISEASE CHARACTERISTICS}

1.1 Name of the disease (synonyms)

$6 q 24$ Transient Neonatal Diabetes Mellitus (6q24 TNDM or TNDM1); Diabetes Mellitus, Transient Neonatal (TND, DMTN); Imprinted transient neonatal diabetes (iTND).

\subsection{OMIM\# of the disease}

601410 .

1.3 Name of the analysed genes or DNA/Chromosome segments 6q24; PLAGL1 Imprinting control region (ICR); ZFP57.

1.4 OMIM\# of the gene(s)

PLAGL1, 603044; HYMAI, 606546; ZFP57, 612192.

1.5 Mutational spectrum ${ }^{1,2}$

UPD(6q24)pat a,b

Paternally inherited duplication of PLAGL ${ }^{\mathrm{C}}$

PLAGLI ICR hypomethylation

aOwing to very small patient numbers, the precise percentages of different molecular aetiologies differ slightly as the sizes of patient cohorts increase.

$>90 \%$ of paternal uniparental disomy in 6q24 TNDM is whole-chromosome isodisomy; however, segmental UPD and heterodisomy are also seen. Maternal uniparental disomy of chromosome 6 is not associated with 6q24 TNDM.

CPaternally inherited duplications of varying sizes have been identified in 6q24 TNDM, but as all contain PLAGL1, they are here designated PLAGL1 duplications. Maternally inherited

duplications of PLAGL1 are not associated with 6q24 TNDM.

d $60 \%$ of cases with PLAGL1 hypomethylation have Multilocus Methylation Defect, that is, defects in imprinted DNA methylation at multiple imprinted loci (MLMD). Of these, approximately half have homozygous mutation of ZFP573 (a mutation database exists for ZFP57: www.lovd.nl/ ZFP57). PLAGL 1 hypomethylation may also be part of MLMD associated with rare mutations in NLRP2 (NM_001174081), NLRP7 (NM_001127255) and KHDC3L (NM_001017361), but individuals affected by such syndromes may not present with 6q24 TNDM.

1.6 Analytical methods

DNA methylation analysis can be performed by ASMM RTQ-PCR; MS-MLPA (SALSA kit ME032 (MRC-Holland, Amsterdam, The Netherlands)); MS-PCR, bisulphite pyrosequencing, MS-SnuPE and methylation array. ${ }^{3-7}$ Copy number imbalance can be detected by MS-MLPA, short tandem repeat marker typing and molecular karyotyping (SNP array, aCGH), and in the case of rare large duplications, FISH or cytogenetic analysis. Uniparental disomy analysis can be performed by short tandem repeat marker typing or by molecular karyotyping using SNP array (UPD testing should preferentially include the parents for full informativity).

\subsection{Analytical validation}

Parallel analysis of negative (unaffected) and positive (affected) controls. Determination of methylation and copy number reference ranges in unaffected individuals (for methylation of same tissue type). For methylation analysis fully (in vitro) methylated and unmethylated (eg, whole-genome amplification) controls should be included.

1.8 Estimated frequency of the disease $1: 300000 .^{8}$

\subsection{Diagnostic setting}

\begin{tabular}{lll}
\hline & Yes & No \\
A. (Differential) diagnostics & $\bigotimes$ & $\square$ \\
B. Predictive testing & $\bigotimes$ & $\square$ \\
C. Risk assessment in relatives & $\bigotimes$ & $\square$ \\
D. Prenatal & $\bigotimes$ & $\square$ \\
\hline
\end{tabular}

Comment:

Prenatal testing may in principle be requested, in cases of familial chromosomal rearrangements potentially affecting the copy number of PLAGL1 or leading to UPD of this region, as well as in cases of familial ZFP57 mutations. In practice, the rarity of the disorder makes prenatal testing extremely rare. In principle, prenatal testing for genomic disturbances (duplications, point mutations) can be offered without limitations and can support clear genetic counselling. Prenatal methylation-specific testing is not common, due to insufficient knowledge about the prenatal setting of the PLAGL1 imprinting mark. Special consideration of recurrence risk is required for the rare maternal effect mutations in NLRP2, NLRP7 or KHDC3L (see Section 3.4).

\subsection{Analytical sensitivity}

(proportion of positive tests if the genotype is present to the best of our knowledge at the moment given that the condition is so rare)

\section{UPD(6q24)pat}

$>95 \%$

Paternal duplication of $6 q 24$

$>95 \%$

PLAGL1 ICR hypomethylation

$>95 \%$ *

ZFP57 mutation

$>95 \%$

*Note that mosaicism is rare in cases presenting with 6q24 TNDM tested so far.

\subsection{Analytical specificity}

(proportion of negative tests if the genotype is not present) Nearly $100 \%$.

\footnotetext{
${ }^{1}$ Faculty of Medicine, University of Southampton, Southampton, UK; ${ }^{2}$ Wessex Regional Genetics Laboratory, Salisbury Health Care Trust, Salisbury, UK; ${ }^{3}$ Institute of Human Genetics, Christian-Albrechts-University Kiel, University Hospital Schleswig-Holstein, Campus Kiel, Schwanenweg 24, Kiel, Germany; ${ }^{4}$ Molecular (Epi)Genetics Laboratory, Research Unit, Hospital Universitario Araba, Vitoria-Gasteiz, Alava, Spain; 5 Wessex Clinical Genetics Service, Princess Anne Hospital, Coxford Road, Southampton, UK *Correspondence: Dr D Mackay, Wessex Regional Genetics Laboratory, Salisbury District Hospital, Salisbury SP2 8BJ, UK. Tel: +44 1722 425048; Fax: + 44 1722 331531; E-mail: djgm@soton.ac.uk
} 


\subsection{Clinical sensitivity}

(proportion of positive tests if the disease is present)

70-80\%.

Note that neonatal diabetes is genetically heterogeneous and, besides the 6q24-linked form described in this gene card, includes other monogenetic forms. ${ }^{9-11}$ Mutations of ABCC8 (MIM\#600509) and KCNJ11 (MIM\#600937) account for 30\% of TNDM, but have a distinct clinical history, with less-extreme low birthweight, and later onset and remission. ${ }^{2,10}$ Moreover, forms of permanent NDM exist. If these monogenetic forms being part of the differential diagnoses are not considered, the sensitivity (ie, 6q24 aberration detected by test if the disease is present) is estimated to be $70-80 \%$

\subsection{Clinical specificity}

(proportion of negative tests if the disease is not present)

Nearly $100 \%$.

\subsection{Positive clinical predictive value}

(lifetime risk to develop the disease if the test is positive)

A small number of cases ( $n=3$ in a cohort of 163 cases) have been described where individuals with $6 q 24$ TNDM mutations did not present neonatally, but subsequently, with disorders such as insulin resistance or gestational diabetes. ${ }^{12,13}$ Owing to the rarity of the disorder, the lifetime risk of later presentation in these individuals is not established.

Note that diabetes presenting at birth resolves in the first few months of life. Earlier clinical studies indicated that $\sim 50 \%$ of individuals presenting neonatally with $6 q 24$ TNDM would subsequently develop a disorder akin to type 2 diabetes, in later childhood or adulthood, ${ }^{14}$ but there is no definitive research to confirm this.

\subsection{Negative clinical predictive value}

(Probability not to develop the disease if the test is negative)

Index case in that family had been tested:

Approaching 100\%, where the proband has a positive diagnosis of a $6 q 24$ anomaly.

Index case in a family that has not been tested:

$<80 \%$.

Where a proband has a clinical diagnosis of transient neonatal diabetes but no molecular diagnosis has been performed, then the molecular cause may be $6 q 24$ aberration, or mutation in ABCC8/ KIR6.2. In a hyperglycaemic infant under 6 months of age, molecular diagnoses of both transient and permanent neonatal diabetes should be considered. ${ }^{9}$

\section{CLINICAL UTILITY}

3.1 (Differential) diagnostics: The tested person is clinically affected

(To be answered if in 1.9 'A' was marked)

\subsubsection{Can a diagnosis be made other than through a genetic test?}

\begin{tabular}{|c|c|c|}
\hline No & $\square$ (continue with 3.1 .4 ) & \\
\hline \multirow[t]{7}{*}{ Yes } & 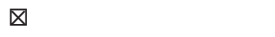 & \\
\hline & Clinically & $凶$ \\
\hline & Imaging & $\square$ \\
\hline & Endoscopy & $\square$ \\
\hline & Biochemistry & $\nabla^{*}$ \\
\hline & Electrophysiology & $\square$ \\
\hline & Other (please describe) & \\
\hline
\end{tabular}

3.1.2 Describe the burden of alternative diagnostic methods to the patient

A diagnosis of neonatal diabetes can be made clinically in infants under 6 months of age, with combined biochemical and immunological (absence of antibodies, no HLA-association) analysis; however, genetic diagnosis is warranted for differentiating 6q24 TNDM from other (monogenic) causes of TNDM where clinical history and management are different. ${ }^{9}$ Moreover, molecular testing enables differential diagnosis at manifestation (when the transient nature is not known) between transient and permanent neonatal diabetes.

\subsubsection{How is the cost effectiveness of alternative diagnostic methods} to be judged?

Classifying 6q24 TNDM is useful for targeting appropriate management of neonatal hyperglycaemia., ${ }^{9,15}$

\subsubsection{Will disease management be influenced by the result of a genetic test?}

\section{No $\square$ \\ Yes $\otimes$ \\ Therapy \\ (please}

describe)

Prognosis (please

describe)
Rapid and effective clinical management is essential for neonatal hyperglycaemia. However, 6q24 TNDM, as its name implies, is transient, usually not combined with ketoacidosis, and typically remitting within the first months of life. 6q24 TNDM is normally managed with rehydration and exogenous insulin therapy, while other monogenic causes of neonatal diabetes (eg, permanent (OMIM\#606176) and transient (OMIM\#610582) neonatal diabetes caused by potassium channel mutations) may be managed by sulphonylurea therapy instead. ${ }^{12,15}$

Making a genetic diagnosis means that the childhood prognosis is positive. Most infants with 6q24 TNDM remit before 4 months of age and require no further immediate treatment. In the longer term, the risk of developing non insulin-dependent diabetes was estimated at $50 \% .{ }^{14}$ It is not clear which patients are at risk of relapse, except that metabolic stress such as puberty, pregnancy or intercurrent illness have been identified in some cases. Growth is usually normal by 2 years of age. ${ }^{16}$

The choice of method of treatment with insulin in the neonatal phase would be influenced by genetic testing. In particular, an insulin pump is an apt choice of treatment for permanent NDM, but not so for TNDM given that it is very likely to remit after a few months. Moreover, treatment of adolescents/adults in the relapse phase that occurs in many patients will be influenced by genetic testing, given that a few reports have suggested these patients later in life respond well to oral therapies, at least initially, and do not necessarily need insulin. Some 6q24 TNDM patients have additional clinical features, such as macroglossia, abdominal anomalies, heart defects or developmental delay, and these may modify prognosis. ${ }^{2,13}$ Sometimes this is due to

multi-locus methylation defect, which in some cases is associated with an increased number of additional clinical features including developmental delay. ${ }^{2,13}$

In 6q24 TNDM due to gross chromosomal imbalances the size and gene content of the imbalanced region(s) might determine a more unfavourable prognosis particularly regarding nondiabetic phenotypes. ${ }^{14}$

In a cohort of 43 children with 6q24 TNDM, 6 patients became clinically hypoglycaemic after diabetes remission, and required ongoing treatment. ${ }^{17}$ Because of this, clinicians may wish to advise continued blood glucose monitoring for the possibility of 
hypoglycaemia in the months after initial remission of the diabetes.

Management Neonatal diabetes should be managed acutely according to

(please

describe) paediatric endocrinology guidelines. ${ }^{9,15}$ Additional clinical features and congenital abnormalities are present in a minority of cases, eg, macroglossia ( $44 \%$ of cases), umbilical hernia (21\%) and less frequent findings, and should be managed appropriately. Note there is no report of macroglossia requiring surgical intervention in this disorder.
3.2 Predictive setting: The tested person is clinically unaffected but carries an increased risk based on family history

(To be answered if in 1.9 'B' was marked)

\subsubsection{Will the result of a genetic test influence lifestyle and prevention?}

If the test result is positive (please describe):

Yes.

If the test result is positive, there is an $\sim 50 \%$ risk of non-insulindependent diabetes developing in adolescence or adulthood. The predisposing factors are not fully understood, but diabetes may be precipitated by metabolic stresses such as puberty, pregnancy, illness or predisposing lifestyle factors. Therefore, those very rare individuals with a positive test result but without neonatal presentation (Section 2.5) should be vigilant for signs of incipient diabetes.

If the test result is negative (please describe):

No

3.2.2 Which options in view of lifestyle and prevention does a person at-risk have if no genetic test has been done (please describe)?

(i) In a pedigree with an intrachromosomal duplication of PLAGL1, a male has a $50 \%$ risk of transmitting a duplicated allele to offspring, whether his own duplication was maternally or paternally transmitted. In case of interchromosomal changes leading to gain of $6 q 24$ the segregation might be dependent on the size of the exchanged fragments. Carriers of a balanced translocation affecting chromosome 6 have an increased risk for their offspring carrying an unbalanced aberration of $6 q 24$, which in case of paternal gain leads to $6 q 24$ TNDM. Similarly, there is theoretically an increased risk for $\mathrm{UPD}(6 q 24)$ pat due to trisomy/monosomy rescue. (ii) In a pedigree with ZFP57 mutation, homozygous individuals are at risk of $6 q 24$ TNDM.

3.3 Genetic risk assessment in family members of a diseased person Most molecular aberrations in 6q24 TNDM are sporadic (UPD(6q24)pat and the majority of PLAGL1 hypomethylation cases). ${ }^{1}$ In these cases, family members are at only population risk. However, for chromosomal aberrations leading to duplication of $6 q 24$ and ZFP57 mutation, there is a risk to family members. Homozygous mutations of ZFP57 and paternal inheritance of $6 q 24$ duplication both carry in principle $100 \%$ risk of $6 q 24$ TNDM (though nonpenetrance is observed, see earlier). Also balanced chromosome 6 aberrations in one of the parents lead to an increased risk for $6 q 24$ TNDM as part of gain affecting $6 q 24$ or UPD derived from malsegregation and rescue, respectively. In patients with MLMD, maternal effect mutations might lead to an up to $100 \%$ recurrence risk in offspring.

\footnotetext{
3.3.1 Does the result of a genetic test resolve the genetic situation in that family?

Yes.
}

3.3.2 Can a genetic test in the index patient save genetic or other tests in family members?

Yes.

\subsubsection{Does a positive genetic test result in the index patient enable a} predictive test in a family member?

Yes.

\subsection{Prenatal diagnosis}

(To be answered if in 1.9 ' $\mathrm{D}$ ' was marked)

\subsubsection{Does a positive genetic test result in the index patient enable a prenatal diagnosis?}

Yes, although see caveat regarding methylation testing. However, due to the rarity of the disorder information is very scarce at present.

\section{IF APPLICABLE, FURTHER CONSEQUENCES OF TESTING}

Please assume that the result of a genetic test has no immediate medical consequences. Is there any evidence that a genetic test is nevertheless useful for the patient or his/her relatives? (Please describe)

The identification of a mutation or epimutation allows delineation of recurrence risk for the patient and his or her family as well as indicating risk of diabetes recurrence in later life.

\section{CONFLICT OF INTEREST}

The authors declare no conflict of interest.

\section{ACKNOWLEDGEMENTS}

This work was supported by EuroGentest2 (Unit 2: 'Genetic testing as part of health care'), a Coordination Action under FP7 (Grant Agreement Number 261469) and the European Society of Human Genetics. The authors are members of the COST Action BM1208. DJGM and IKT are supported by Diabetes UK, MRC and NIHR; SB and RS are supported by BMBF (Ministry of Education and Science) in the framework of the consortium 'Diseases caused by imprinting defects: clinical spectrum and pathogenetic mechanisms' (FKZ: 01GM0886 and 01GM1114); GPN is partially funded by the I3SNS Program of the Spanish Ministry of Health (CP03/0064; SIVI 1395/09).

1 Temple IK, Mackay DJG, Docherty LE: Diabetes mellitus, 6q24-related transient neonatal. In: Pagon RA, Adam MP, Bird TD et al: (eds.) GeneReviews ${ }^{\mathrm{TM}}$, (initial posting: 10 Oct 2005; updated 27 Sep 2012) University of Washington: Seattle, WA, USA, 1993-2013.

2 Docherty LE, Kabwama S, Lehmann A et al: 6q24 Transient Neonatal Diabetes Mellitus (6q24 TNDM)-clinical presentation and genotype phenotype correlation in an international cohort of cases. Diabetologia 2013; 56: 758-762.

3 Mackay DJ, Callaway JL, Marks SM et al: Hypomethylation of multiple imprinted loci in individuals with transient neonatal diabetes is associated with mutations in ZFP57. Nat Genet 2008; 40: 949-951.

4 Maupetit-Mehouas S, Azzi S, Steunou V et al: Simultaneous hyper- and hypomethylation at imprinted loci in a subset of patients with GNAS Epimutations underlies a complex and different mechanism of multilocus methylation defect in pseudohypoparathyroidism type 1b. Hum Mutat 2013; 34: 1172-1180.

5 Metz C, Cavé H, Bertrand AM et al: Neonatal diabetes mellitus: chromosomal analysis in transient and permanent cases. J Pediatr 2002; 141: 483-489.

6 Begemann M, Leisten I, Soellner L et al: Use of multilocus methylation-specific single nucleotide primer extension (MS-SNuPE) technology in diagnostic testing for human imprinted loci. Epigenetics 2012; 7: 473-481.

7 Martin-Subero JI, Bibikova M, Mackay D et al: Microarray-based DNA methylation analysis of imprinted loci in a patient with transient neonatal diabetes mellitus. Am J Med Genet A 2008; 146A: 3227-3229.

8 Wiedemann B, Schober E, Waldhoer T et al: Incidence of neonatal diabetes in Austriacalculation based on the Austrian Diabetes Register. Pediatr Diabetes 2010; 11 : 18-23.

9 Hattersley A, Bruining J, Shield J et al: The diagnosis and management of monogenic diabetes in children and adolescents. Pediatr Diabetes 2009; 10: S33-S42. 
10 Polak M, Cavé H: Neonatal diabetes mellitus: a disease linked to multiple mechan isms. Orphanet J Rare Dis 2007; 2: 12.

11 Flanagan SE, Patch A, Mackay DJG et al: Mutations in KATP channel genes cause transient neonatal diabetes and permanent diabetes in childhood or adulthood Diabetes 2007; 56: 1930-1937.

12 Valerio G, Franzese A, Salerno M et al: Beta-cell dysfunction in classic transient neonatal diabetes is characterized by impaired insulin response to glucose but normal response to glucagon. Diabetes Care 2004; 27: 2405-2408.

13 Boonen SE, Mackay DJ, Hahnemann JM et al: Transient neonatal diabetes, ZFP57 and hypomethylation of multiple imprinted loci: a detailed follow-up. Diabetes Care 2013, 36: 505-512.
14 Temple IK, Gardner RJ, Mackay DJ et al: Transient neonatal diabetes: widening the understanding of the etiopathogenesis of diabetes. Diabetes 2000; 49: 1359-1366.

15 Støy J, Greeley SA, Paz VP et al: United States Neonatal Diabetes Working Group. Diagnosis and treatment of neonatal diabetes: a United States experience. Pediatr Diabetes 2008; 9: 450-459.

16 Shield JP, Temple IK, Sabin M et al: An assessment of pancreatic endocrine function and insulin sensitivity in patients with transient neonatal diabetes in remission. Arch Dis Child Fetal Neonatal Ed 2004; 89: F341-F343.

17 Flanagan SE, Mackay DJ, Greeley SA et al: Hypoglycaemia following diabetes remission in patients with $6 \mathrm{q} 24$ methylation defects: expanding the clinical phenotype. Diabetologia 2013; 56: 218-221. 